\title{
Recognising attention deficit hyperactivity disorder across the lifespan
}

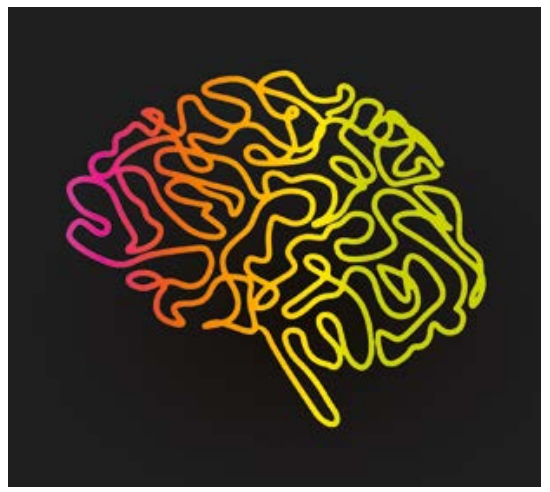

CPD

\section{Alison Poulton}

\section{Background}

Attention deficit hyperactivity disorder (ADHD) is the most common neurodevelopmental disorder and is estimated to affect approximately 10\% of children and $5 \%$ of adults. It is associated with impaired functioning. Recognition and appropriate treatment can make a substantial difference.

\section{Objective}

The aim of this article is to provide a framework for recognising ADHD and the ways that it can present at different stages of the lifespan.

\section{Discussion}

General practitioners have a vital role in identifying people who may have undiagnosed ADHD. It is important to take a history targeted at eliciting problems in everyday functioning. People who are intellectually able may have developed coping strategies that mask their ADHD; in those with other psychiatric diagnoses, the underlying ADHD may be overlooked. Timely and effective treatment can make an enormous difference to the individual and to their family and peers and can reduce the likelihood of subsequent development of secondary mental health and social issues.
ATTENTION DEFICIT HYPERACTIVITY DISORDER (ADHD) is normally framed as a categorical diagnosis - either someone has it, or they do not. In fact, it is better considered as a spectrum, such that everyone shows some of the features to a greater or lesser extent. ADHD is inherited, but the genetics are complex, with many genes making small contributions to the final phenotype. ${ }^{1}$ Environmental stressors such as premature birth may also contribute. ${ }^{2}$

The features of ADHD include inattention, hyperactivity and impulsivity (Table 1$).{ }^{3}$ People with ADHD may show the full range of features (combined-type ADHD) or may just have inattention (inattentive ADHD). Oppositional defiant disorder (ODD) is commonly associated with ADHD, such that $40 \%$ of primary school children with ADHD also have diagnosable ODD, ${ }^{4}$ and it is likely that another $40 \%$ show clinically significant features of ODD but do not reach the diagnostic threshold. Therefore, ODD may be considered as part of the presentation of ADHD in a large proportion of affected children and adolescents. ${ }^{5}$ ODD behaviour tends to be most prominent in the preschool age group ('terrible twos') and in adolescence.

ADHD cannot be diagnosed simply in terms of fulfilling a certain number of listed criteria. The key to diagnosis is that the symptoms are associated with functional impairment (Box 1) ${ }^{6}$ This should be across two or more settings, usually home and either at work, school or preschool.

Functional impairment may be defined as a person being unable to meet the expectations of their roles or commitments. A person with severe ADHD may meet diagnostic criteria throughout their life, while others only become diagnosable later as their responsibilities exceed their ability to concentrate and organise their life. Therefore, the assessment should evaluate the person's ability to carry out age-appropriate functions.

People with ADHD can be helped substantially. Treatment consists of psychological intervention, often in combination with medication.

\section{Preschool children}

Preschool children are often active and impulsive and may not sit and concentrate for long. Despite this, most preschoolers can concentrate sufficiently to develop age-appropriate skills, and the behavioural functioning of most improves substantially as they approach school age. There is a real danger of overdiagnosing ADHD, particularly when using diagnostic ratings that are standardised for school-aged 
children. However, ADHD should be considered in children whose behaviour is extreme, as the children with the most severe problems tend to present at a younger age.

It is important to assess a child's functioning in two different settings. Enrolling a child into preschool will clarify whether he/she is able to function better there than at home, as might be the case in some family circumstances.

The children with ADHD who are most impaired are usually those who also have severe ODD. ${ }^{7}$ These children may have frequent, severe temper outbursts. Their impulsive, unpredictable behaviour associated with anger and aggression may make them unmanageable in preschool; they may present with safety issues, such as running out onto a road. They may be unmanageable when shopping. Parents (or other carers) may be stressed because of a child's frequent, unpredictable and violent temper outbursts.

Psychological intervention in preschoolers involves teaching the parents strategies for managing the child, which can be very effective. Most paediatricians would be reluctant to treat preschoolers with medication. However, in extreme cases, it can be helpful. ${ }^{8}$

\section{Primary school children}

The peak age of diagnosis of ADHD is in primary school children aged $5-10$ years. ${ }^{7}$ These children typically present with poor concentration that affects their learning. A child who is unable to concentrate for long may become bored and disruptive.
Teachers are usually very good at identifying ADHD in primary school children. However, children who are not hyperactive may attract less attention.

ADHD can affect peer relationships. A child who has difficulty listening and concentrating may appear bossy and controlling. Children with ODD may develop a reputation for being aggressive or may be targeted by others and provoked into losing their temper. They may therefore be either the victim or perpetrator of bullying. As children progress through school, they expect more from their friends, and a child who does not listen and respond appropriately may be excluded.

ODD can mask ADHD: if a child

habitually refuses to attempt any schoolwork, the teacher may not know that they have difficulty concentrating. ODD is unusual without ADHD, and a pattern of behavioural escalation to avoid work may point towards underlying difficulty concentrating.

Functional impairment at home may manifest as disorganisation requiring numerous reminders for getting dressed and ready. The concentration span can be evaluated by asking the parent whether homework takes far longer and requires more parental input than it should. Children with ADHD may have difficulty persisting with activities that involve mental effort, complaining of boredom unless they have electronic devices that provide constant stimulation and reward.

Intervention may involve additional learning support, together with behavioural strategies at school and at home that have clear, realistic expectations

Table 1. Features of attention deficit hyperactivity disorder and oppositional defiant disorder

\begin{tabular}{lc} 
Attention deficit hyperactivity disorder & Oppositional defiant disorder \\
\hline $\begin{array}{l}\text { Inattention - Unable to concentrate or listen } \\
\text { for long, moving quickly from one activity to } \\
\text { another, bored easily }\end{array}$ & $\begin{array}{l}\text { Negative attitude, hostile, aggressive - } \\
\text { temper outbursts, bullying } \\
\text { - Hyperactivity - Constant, restless activity, } \\
\text { with difficulty sitting still, climbing, running } \\
\text { off, excessively talkative }\end{array}$ \\
- Impulsivity - Quick reactions, not having \\
time to think and make a decision
\end{tabular}

\section{Box 1. Modalities of functional} impairment

- Is considered capable of higher achievement - could/should do better

- Behaviour presents unreasonable stress or disruption in school/work

- Behaviour presents unreasonable stress or disruption in the family

- Behaviour significantly affects peer relationships

- Person is aware of having difficulties and has low self-esteem 
advantage of the greater sophistication that comes with maturity. It can be helpful to ask the adolescent what percentage of class time they are actually listening or concentrating. This estimate may be enlightening and may help to identify quietly inattentive children.

\section{Adults}

The recognition of ADHD in childhood has greatly improved over the past 20 years, but there are adults whose diagnosis was missed and who may only realise that they have ADHD when their children are diagnosed. As the demands of life increase, so does the scope for dysfunction. A woman who escaped diagnosis in childhood (she may have been a quiet daydreamer) may struggle to cope with the complexities of holding a job, raising children and running a household.

When a common condition is under-recognised, it will tend to be the patients with more severe cases (ie those with comorbid psychiatric diagnoses) who are identified. The lifetime of underachievement, disorganisation and impulsive behaviour and associated poor self-esteem may progress to anxiety, depression or substance abuse. Adults with such mental health conditions should be screened, because neglecting the underlying ADHD reduces treatment efficacy. It is also important to consider ADHD in people who may appear to be functioning in society but, on closer questioning, are experiencing stress due to their inefficiency and disorganisation. People whose career choices involve high-order cognitive functioning may reach a stage where their previously undiagnosed ADHD starts to hold them back. Such people may have well-developed strategies for overcoming the challenges of ADHD, so that their impairment may not be immediately evident. The lines of questioning suggested in Table 2 may be helpful, particularly a description of the pattern of concentration over the course of the working day.

ADHD is likely to be most under-diagnosed in the elderly but may cause increasing problems as cognitive decline erodes a person's coping mechanisms. ADHD may become evident on the illness or death of a partner who has been doing all the organisation and decision making. Therefore, it is important to consider ADHD, particularly in those with a family history.

Other conditions may resemble or co-exist with ADHD (Table 3).

Adults with ADHD may benefit from psychological intervention for anger management, relationship counselling or an ADHD coach to help them develop life skills and strategies for managing their ADHD. Medication can also be highly beneficial for stabilising the mood and improving the concentration.

\section{Table 2. Diagnosing attention deficit hyperactivity disorder}

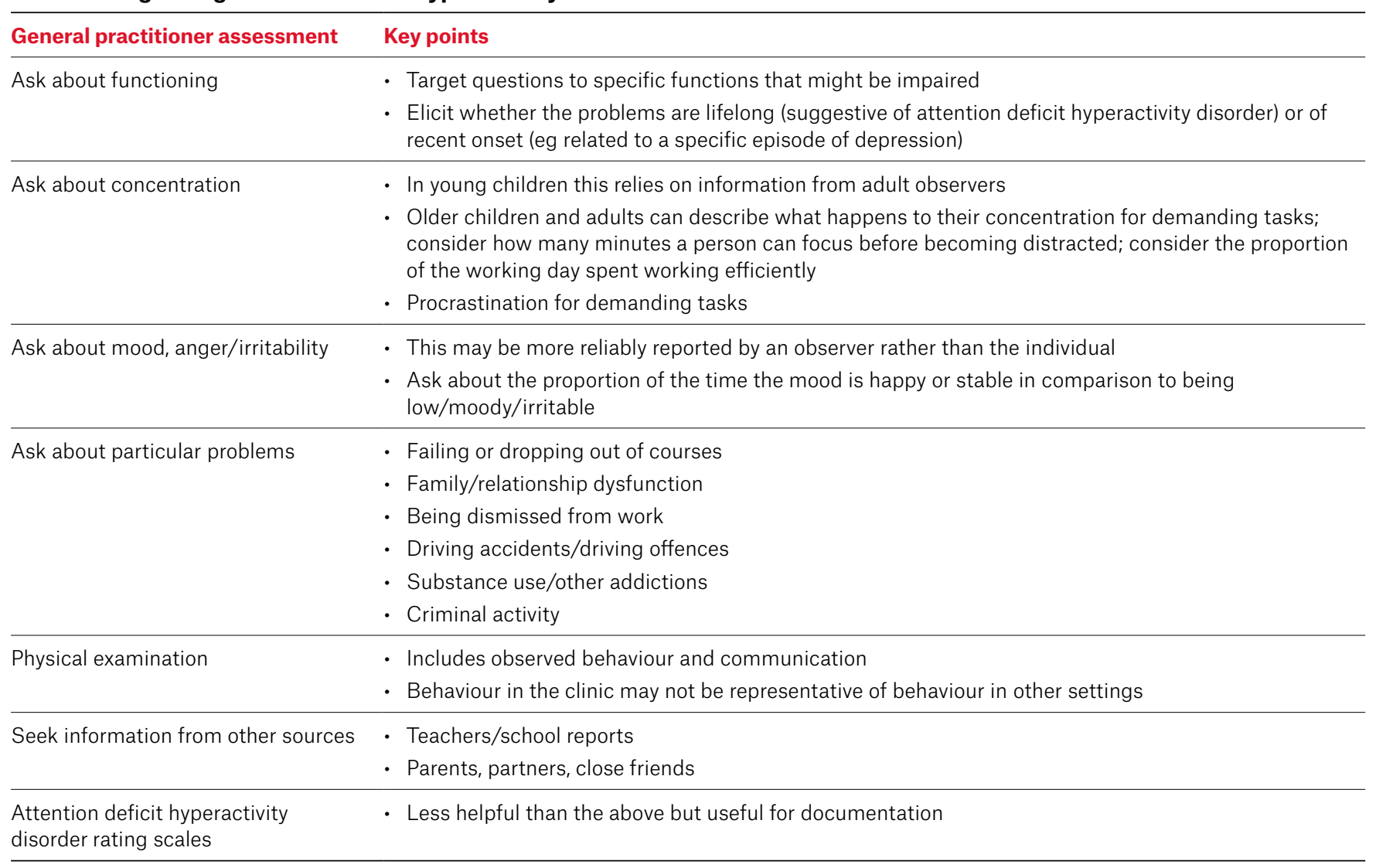




\section{Table 3. Differential diagnosis - conditions that often co-exist with attention deficit hyperactivity disorder*}

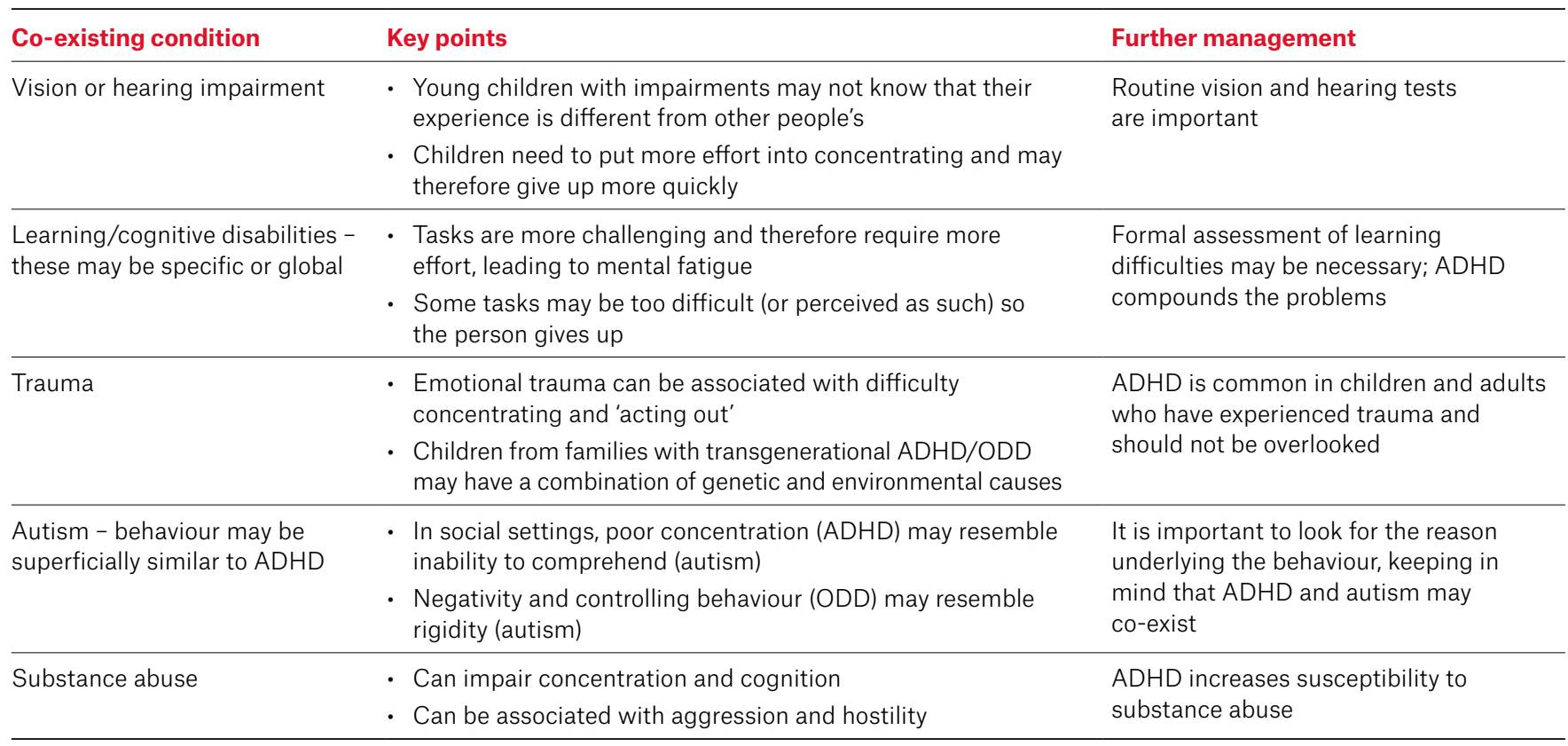

*When considering disability, it is important to assess the person's highest level of functioning. A person who lacks skills or comprehension is always limited by their disability. By contrast performance in ADHD is task dependent and variable from one day to another (even if 'good days' are infrequent). $A D H D$, attention deficit hyperactivity disorder; $O D D$, oppositional defiant disorder

\section{Conclusion}

ADHD is common at all ages. GPs have a vital role in identifying people whose functioning may be impaired because of ADHD. This is particularly important when assessing people with less obvious symptoms who might otherwise remain undiagnosed. Timely and effective treatment can make an enormous difference to the individual and to their family and peers and can reduce the likelihood of subsequent development of secondary mental health issues.

\section{Key points}

- ADHD is an inherited condition and is common at all ages.

- ADHD is often undiagnosed, particularly in adults.

- Treatment can be highly effective for improving functioning.

- For most patients, treatment involves a combination of psychological and pharmacological interventions.

- Psychological interventions should be started as early as possible and do not require a formal diagnosis.

- Medication requires specialist treatment.

\section{Author}

Alison Poulton MBBChir, MD (Cantab), FRACP, Senior Lecturer in Paediatrics, Brain Mind Centre Nepean, University of Sydney, NSW

Competing interests: None.

Funding: AP reports personal fees and non-financial support from Shire/Takeda, outside the submitted work. Provenance and peer review: Commissioned, externally peer reviewed.

Correspondence to: alison.poulton@sydney.edu.au

\section{References}

1. Faraone SV, Larsson H. Genetics of attention deficit hyperactivity disorder. Mol Psychiatry 2019;24(4):562-75. doi: 10.1038/s41380-0180070-0.

2. Thapar A, Cooper M. Attention deficit hyperactivity disorder. Lancet 2016;387(10024):1240-50. doi: 10.1016/S0140-6736(15)00238-X.

3. American Psychiatric Association. Diagnostic and statistical manual of mental disorders (DSM-5). 5th edn. Arlington, VA: APA, 2013.

4. MTA Cooperative Group. A 14-month randomized clinical trial of treatment strategies for attention-deficit/hyperactivity disorder. The MTA Cooperative Group. Multimodal treatment study of children with ADHD. Arch Gen Psychiatry 1999;56(12):1073-86. doi: 10.1001/ archpsyc.56.12.1073.

5. Poulton A, Nanan R. The attention deficit hyperactivity disorder phenotype as a summation of deficits in executive functioning and reward sensitivity: Does this explain its relationship with oppositional defiant disorder? Australas Psychiatry 2014;22(2):174-78. doi: 10.1177/1039856213517949.
6. Poulton A. Explanation sheets. ADHD: Patient explanation. Sydney, NSW: Poulton ADHD, 2019. Available at www.poultonadhd.com.au/ explanationsheets.html [Accessed 15 January 2021].

7. Eysbouts Y, Poulton A, Salmelainen P. Stimulant medication in preschool children in New South Wales. J Paediatr Child Health 2011;47(12):870-74. doi: 10.1111/j.1440-1754.2011.02107.x.

8. Greenhill L, Kollins S, Abikoff H, et al. Efficacy and safety of immediate-release methylphenidate treatment for preschoolers with ADHD. J Am Acad Child Adolesc Psychiatry 2006;45(11):1284-93. doi: 10.1097/01.chi.0000235077.32661.61.

9. Ross L, Sapre V, Stanislaus C, Poulton AS. Dose adjustment of stimulants for children with attention-deficit/hyperactivity disorder: A retrospective chart review of the impact of exceeding recommended doses. CNS Drugs 2020;34(6):643-49. doi: 10.1007/s40263-02000725-5.

10. Matthies S, Holzner S, Feige B, et al. ADHD as a serious risk factor for early smoking and nicotine dependence in adulthood. J Atten Disord 2013;17(3):176-86. doi: 10.1177/1087054711428739.

correspondence ajgp@racgp.org.au 